\title{
Universidades Comunitarias en Brasil: ¿Por qué hay que Perfeccionar su Modelo de Gestión?
}

\author{
Paulo Fossatti* y Luiz C. Danesi \\ Universidad La Salle, Avenida Victor Barreto, 2288, Canoas, Brasil (e-mail: paulo.fossatti@unilasalle.edu.br; \\ luizcarlosd690@gmail.com)
}

${ }^{*}$ Autor a quien debe ser dirigida la correspondencia

Recibido Ene. 23, 2018; Aceptado Abr. 4, 2018; Versión final May. 10, 2018, Publicado Oct. 2018

\begin{abstract}
Resumen
Este artículo resulta del trabajo de un grupo de investigación del Sur de Brasil, como parte de estudio sobre modelos de gestión en Instituciones Comunitarias de Enseñanza Superior (ICES). El objetivo fue problematizar desafíos de esas instituciones en cuanto al perfeccionamiento de la gestión, en un escenario competitivo, espectro regulatorio gubernamental y carencia de recursos públicos. Se trata de una investigación cualitativa y exploratoria, con datos de reciente estudio de casos múltiples con gestores de ICES y revisión de literatura. Los resultados enuncian acciones institucionales que contribuyen al perfeccionamiento de los modelos de gestión: i) iniciativas exitosas en la adecuación de estructuras y procesos; ii) frágil política de comunicación con el mercado y comunidad externa e interna; iii) buena relación con autoridades gubernamentales y segmentos económicos, empresariales y sociales; y iv) articulaciones políticas y actitudes emprendedoras e innovadoras. Se concluye sobre la necesidad de continuar explotando buenos indicadores de gestión y nuevas formas de gobernanza universitaria.
\end{abstract}

Palabras clave: modelos de gestión; universidades comunitarias; enseñanza superior; buenas prácticas de gestión; gobernanza universitaria

\section{Community Universities in Brazil: Why do you Need to Improve your Management Model?}

\begin{abstract}
The article is the result of the work of a research group from Southern Brazil, as part of a study on management models in Community Institutions of Higher Education (ClHE). It aims to problematize challenges of these institutions in relation to the improvement of management in a competitive scenario, governmental regulatory and lack of public resources. It is a qualitative and exploratory research with data from recent multiple case studies with CIHE managers and literature review. The results enunciate institutional actions that contribute to the improvement of the management models: i) successful initiatives in the adequacy of structures and processes; ii) fragile policy of communication with the market and the external and internal community; iii) good relationship with governmental authorities, business, economic and social segments; and iv) political articulations, entrepreneurial and innovative attitudes. It is concluded on the necessity of continuing exploring good management indicators and new forms of university governance.
\end{abstract}

Keywords: management models; community universities; higher education; good management practices; university governance 


\section{INTRODUCCIÓN}

La temática de esta investigación es la gestión universitaria. El estudio está inserido en la línea de investigación Gestión, Educación y Políticas Públicas de un Programa de Posgrado en Educación de una Universidad Comunitaria al Sur de Brasil. Tiene como objetivo problematizar los desafíos de las Instituciones Comunitarias de Enseñanza Superior (ICES) de Brasil en cuanto al perfeccionamiento de los modelos de gestión, en un escenario de fuerte competitividad, amplio espectro regulatorio gubernamental y carencia de recursos públicos para la educación. Se trata de un trabajo cualitativo y exploratorio de estudio de casos múltiples con gestores de ICES y revisión de literatura pertinente. La contemporaneidad se caracteriza por un ambiente de continuos y rápidos cambios. Las reflexiones de Morgan (2011) posibilitan partir desde una retrospectiva de los procesos estructurados en las organizaciones y enfocar intereses relevantes para mejorar el desempeño institucional. Según explica el autor, las organizaciones son sistemas abiertos que necesitan ser administrados con extremo cuidado para satisfacer el equilibrio de las necesidades internas y asimismo adaptarse a las circunstancias del medio. De esa manera, los cambios exigen de los gestores una profunda comprensión de los tipos y formas de esos cambios. Para mantenerse en el ambiente competitivo, la organización necesita presentar un modelo de gestión que entienda los tipos de cambios a los que se deberá someter. Los cambios han envuelto grandes y continuas transformaciones en los ámbitos científico, tecnológico, político, sociocultural y, principalmente, económico (Santos, 2011). Esas transformaciones imponen dificultades que deben ser enfrentadas, pero asimismo traen nuevas oportunidades y alternativas. Para que algunas organizaciones tengan condiciones de acompañar y permanecer en el mercado, es necesario que haya modificaciones paradigmáticas en términos de perspectivas, estrategias y el desarrollo de actitudes emprendedoras y creativas que viabilicen la fidelización de sus clientes y la captación de nuevas oportunidades de mercado (Ganga et al, 2014).

En la educación superior estos cambios implican el desarrollo de modelos de gestión que atiendan a los desafíos de una nueva economía y sociedad con sustentabilidad y que conduzcan la institución hacia un incremento de calidad y de excelencia académica. Entre las modificaciones en el escenario mundial se verifica el creciente ascenso de instituciones privadas de enseñanza conquistando un espacio significativo en el "mercado educacional" (Goetsch y Davis, 2014). Este contexto despierta mayor atención sobre los gestores, principalmente los de Instituciones Comunitarias de Educación Superior (ICES) brasileñas. Así el crecimiento de la competitividad en la oferta de servicios educacionales ha implicado gran contraste con una cultura administrativa de las ICES que todavía estaba pautada en conductas intuitivas. La viabilidad institucional pasó a requerir "foco en el negocio". Se trata, pues, de una contribución de la ciencia de la administración a la ciencia de la educación. Está en curso la diseminación del modelo empresarial de gestión en las instituciones universitarias con sustentabilidad y calidad en los servicios ofrecidos (Moraes, 2006). En este escenario, en los últimos años, muchas empresas, instituciones públicas y la sociedad civil han pasado a adherir a las prácticas sustentables en términos económicos, sociales y ambientales (Hesselbarth y Schaltegger, 2014). De esta manera, la sustentabilidad para las instituciones comunitarias se establece a través de la realización de elementos como: conservación de recursos, ambiente construido, calidad del medio ambiente, igualdad social y participación política (Blowers, 1991). Así, las ICES deben vislumbrar prácticas sustentables en su dimensión académica y asimismo en sus prácticas de gestión.

El campo académico se debe constituir en un espacio propicio para difundir valores sustentables (Alshuwaikhat y Abubakar, 2008), ofreciendo servicios educacionales y de extensión con calidad. De esta forma, contribuye intelectualmente con la sociedad (Foo, 2013), a través de la transformación del conocimiento, cultivo de talentos e innovación (Tan et. al., 2014). En la gestión, las ICES se vienen reorientando hacia prácticas sustentables (Wals, 2014). En muchas de ellas eso está ocurriendo por cuenta de presiones externas de stakeholders como mantenedoras, gobierno, agencias de fomento y mercado (Müller-Christ et. al., 2.014), y del entorno comunitario. De esa forma, la universidad comunitaria atiende, desde hace mucho tiempo, a su razón de ser. Sin embargo, tiene que adecuarse siempre a la evolución de los tiempos. Dentro de este contexto, el objetivo del presente estudio es la problematización de la necesidad de cambios en el modelo tradicional de gestión de las ICES. De ahí viene el cuestionamiento que proponemos ya en el título del trabajo: ¿por qué hay que perfeccionar el modelo de gestión de las ICES en Brasil?

Con relación al camino metodológico, se trata de una investigación cualitativa exploratoria, con características de un estudio de casos múltiples. Recae sobre el cuestionamiento en cuanto al perfeccionamiento del modelo de gestión en la universidad comunitaria brasileña. Tiene como campo empírico las ICES del Sur de Brasil. De acuerdo con Stake (1998), este tipo de estudio se direcciona al "[...] estudio de la particularidad y de la complejidad de un caso singular, para llegar a comprender su actividad en circunstancias importantes". De esta manera, hay que aclarar la inferencia hecha por los autores en esta investigación: a partir del estudio de las ICES del Sur de Brasil, se está infiriendo para las demás universidades comunitarias brasileñas. Así, se han utilizado datos primarios obtenidos recientemente en una 
investigación sobre "Gestión Universitaria Sustentable" conducido por un grupo de investigación de un Programa de Posgrado en Educación de una ICES del Sur de Brasil. En su totalidad, la investigación ha involucrado 14 instituciones, en las cuales se han hecho entrevistas a 54 gestores. En un conjunto de 18 cuestiones, identificamos las manifestaciones asociadas con el modelo de gestión de las ICES. De esta manera, nos ha interesado percibir las implicaciones de las respuestas o aquello que se deduce de ellas.

Esas 14 instituciones están entre las 15 que forman el Consórcio das Universidades Comunitárias Gaúchas (Comung). En total, son 15 las Universidades que componen este órgano, pero una de ellas no ha participado en el estudio. El Comung fue constituido oficialmente el 27 de abril de 1996 y lo integran 15 instituciones de enseñanza superior del estado de Rio Grande do Sul y con 8.735 profesores y más de 11 mil funcionarios, contabiliza a 189.224 estudiantes, constituyéndose como el mayor sistema de educación superior en actuación en Rio Grande do Sul. Se ha llegado al número de 54 gestores aleatoriamente, puesto que se ha enviado la invitación a los rectores de todas las universidades del consorcio, los que han asignado quienes iban a participar.

Se ha utilizado la técnica de Análisis de Contenido (Bardin, 2006) para agrupar, las respuestas de las entrevistas en cuatro categorías, y así propiciar la apuración de los resultados y la discusión con la literatura. Según la autora, las categorías son partes "significativas que permiten la clasificación de los elementos de significación constitutivos del mensaje" (Bardin, 2006). Así, las categorías encontradas fueron: Adecuación de estructuras y procesos; Políticas de comunicación con el mercado, comunidades externa e interna; Relación con autoridades gubernamentales y segmentos económicos, empresariales y sociales; Articulaciones políticas y actitudes emprendedoras e innovadoras.

La arquitectura del artículo trae el desarrollo del tema a continuación, donde buscamos hacer una caracterización de las ICES brasileñas. Acto seguido, en los resultados y discusión, presentamos las representaciones de los participantes en las entrevistas, relacionándolas con la bibliografía consultada. De ahí, surgen nuestras inferencias sobre el tema. Por último, están las consideraciones finales y referencias consultadas.

\section{DESARROLLO DEL TEMA}

Se presentan dos secciones, la primera de ellas relacionada con las universidades comunitarias brasileñas y la segunda, del porqué éstas necesitan un cambio de modelo en su gestión.

\section{¿Quiénes son las universidades comunitarias brasileñas?}

Históricamente, el término universidad gana cuerpo durante el siglo $X V$, cuando profesores y alumnos de corporaciones preexistentes que se habían instituido sin expresa autorización de Rey, Papa, Príncipe o prelado, irradian los modelos de París y Bolonia por el continente europeo (Clark, 2008). La institución universitaria ha retomado las experiencias de las academias de Platón, de la Grecia clásica, pasando del pensamiento mítico a la racionalidad. Con ello, ha posibilitado la transición de la humanidad desde una etapa a la otra. Desde el campo hacia la vida en las ciudades, de los dogmas a la racionalidad, del mundo espiritual al terreno, desde la Edad Media al Renacimiento, ha permitido nuevos tiempos y nuevos paradigmas (Blowers, 1991). En este sentido, la universidad moderna se establece en un contexto temporal e histórico en sus relaciones con el Estado, con el mercado y con las comunidades. Si bien no se concibe trazar leyes y estándares universales de un sistema de enseñanza superior y acerca de sus relaciones con el Estado y la sociedad, se percibe el sostenimiento de la universidad en una matriz moderna, aunque con las naturales divergencias en su identidad. Así, convivimos con sistemas de enseñanza superior basados en mercados, como es el caso de los Estados Unidos, o centralizados en el gobierno, como en China, o entonces centralizados en un proceso de cooperación como Bolonia, en la Comunidad Europea (CE) y sistemas híbridos o de cuasi-mercado como se ha establecido en Brasil (Cascio, 2018).

La educación superior en Brasil nace bajo la égida del Estado. Con la creación de la República por la Constitución de 1891, la Enseñanza Superior es atribuida al poder central, pero no exclusivamente. De esta manera, a lo largo de las décadas, la situación precaria de las universidades públicas brasileñas promovió la discusión alrededor de la escuela pública versus privada (Santos, 2011). El debate continúa y ello se debe a que, en Brasil, las universidades estatales, gratuitas, representan menos del 15\% (Fonif, 2016) de las instituciones de enseñanza superior del país. Para acceder a ellas hay que pasar por un proceso selectivo estricto (si bien en Brasil hay políticas de cuotas para negros, indígenas y pobres). En la escuela básica pasa justo lo contrario: la red estatal, también precarizada, representa la mayoría. Ahora bien, si para entrar a la universidad estatal hay que estar bien preparado, obviamente quienes logran estudiar sin costos son, en su gran mayoría, los hijos de los ricos, que se han preparado en la escuela básica particular. Sin embargo, esta realidad comienza a cambiar a partir de la Ley N. 12.711 , del 29 de agosto de 2012, 
conocida popularmente como la "Ley de las Cuotas" que dispone sobre el ingreso en las universidades federales y en las instituciones federales de enseñanza técnica de nivel medio. En su artículo $1^{\circ}$ afirma que las instituciones federales vinculadas al MEC "reservarán, en cada concurso selectivo para ingreso en los cursos de grado, por curso y turno, como mínimo un 50\% (cincuenta por ciento) de sus vacantes para estudiantes que hayan cursado integralmente la enseñanza media en escuelas públicas" (BRASIL, 2012). Así, el modelo comunitario de enseñanza superior surge por la deficiencia del Estado e incluso ausencia en distintas regiones del país. Santos (2011) señala que en algunos países ya hay una tradición de universidades privadas sin fines de lucro, que con el tiempo han venido a asumir roles semejantes a los de las públicas y acabaron gozando de un estatuto híbrido, entre público y privado (Santos, 2011; 2015).

Entre el fin de la década de los años 1980 y principios de la década de 1990, hubo un incremento de inversiones del sector privado en la educación superior brasileña. El Estado había agotado los recursos presupuestarios para financiar prioritariamente la actividad universitaria en el país. Así, requirió la participación del segmento privado por medio del artículo 209 de la Constitución Federal (Brasil, 1988), reproducido en el artículo $7^{\circ}$ de Ley de Directrices y Bases de la Educación Nacional: "O ensino é livre à iniciativa privada" (Brasil, 1996). En aquella época, las IES se clasificaban en públicas y privadas. La Ley $\mathrm{n}$. 12.881, del 12 de noviembre de 2013 dispone sobre la definición, calificación, prerrogativas y finalidades de las Instituciones Comunitarias de Educación Superior (ICES). Tales universidades nascieron de la iniciativa de sus comunidades, sus Iglesias, sus movimientos sociales. La ley pretendió conferir a esas entidades una calificación distinta, pero que también les garantizase el carácter de institución privada de interés público, actuando en conjunto con el Poder Público en la ampliación de la enseñanza superior. Si bien puedan cobrar mensualidades, las ICES no objetivan lucro y aplican integralmente sus recursos en el mantenimiento de los objetivos institucionales. Son instituciones bastante orientadas al desarrollo local - por ello la concepción de "comunitarias" -, propiciando interacciones y servicios a las comunidades de su alrededor. Además, tienen exenciones fiscales a cambio de becas de estudios que se ofrecen a alumnos con menos condiciones financieras (Brasil, 2013). De esa forma, se han empeñado en la responsabilidad social, con excelencia a través de las actividades de enseñanza, de investigación y de extensión.

Sin embargo, ya a comienzos de la década de 1990, segmentos de la sociedad civil manifestaron apoyo a las Instituciones Comunitarias en Brasil, entre ellos, el Consejo de Rectores de las Universidades Brasileñas (CRUB) (Pinto, 2009). De esta forma, las universidades comunitarias se identifican con la universidad moderna. La universidad es una institución que viene adaptando la evolución de sus prácticas ante a nuevos escenarios resultantes de ambientes dinámicos y de cambios en los contextos económico y social (Baynaghi et. al., 2014.). Son instituciones que perfeccionan sus habilidades en formar profesionales y de realizar investigación científica (Janeiro et. al., 2013).

Las ICES todavía transcienden la esencia común observada en la academia debido a su intensa responsabilidad social comunitaria. Si bien tienen un carácter público, necesitan encargarse de su sustentabilidad con calidad en sus servicios (Moraes, 2006) y diseminar estas responsabilidades en el entendimiento de la cultura universitaria (Too y Bajracharya, 2015). Las ICES, como dicho, tuvieron su reconocimiento oficial como institución comunitaria en 2013, pero siguen dependiendo de mensualidades de alumnos para atender a sus compromisos económicos y financieros. Sin embargo, se abrió un espacio para disputar pliegos de proyectos de investigación en igualdad de condiciones con las instituciones públicas estatales. La tabla 1 ilustra el actual modelo de instituciones de enseñanza superior en Brasil en cuanto a su naturaleza, mantención y sustentabilidad.

Tabla 1: naturaleza de las instituciones de enseñanza superior en Brasil

\begin{tabular}{|c|c|c|}
\hline Naturaleza & Mantenedora & Sustentabilidad \\
\hline $\begin{array}{l}\text { Instituciones Públicas (Art. } 45 \text { de la } \\
\text { Ley de Directrices y Bases de la } \\
\text { Educación Nacional - LDB). }\end{array}$ & $\begin{array}{l}\text { Gobiernos: Federal, Estaduales y } \\
\text { Municipales. }\end{array}$ & $\begin{array}{l}\text { Gratuita y mantenida por el } \\
\text { presupuesto público. }\end{array}$ \\
\hline $\begin{array}{l}\text { Instituciones Privadas (Art. } 45 \text { de la } \\
\text { LDB). }\end{array}$ & $\begin{array}{l}\text { Personas jurídicas o personas } \\
\text { físicas. }\end{array}$ & $\begin{array}{l}\text { Los estudiantes pagan y hay fines } \\
\text { lucrativos. }\end{array}$ \\
\hline $\begin{array}{l}\text { Instituciones Comunitarias } \\
\text { 12.881/2013. }\end{array}$ & $\begin{array}{l}\text { Regionales: } \\
\text { Asociaciones/Fundaciones. } \\
\text { Confesionales: instituciones } \\
\text { religiosas de diversas creencias. }\end{array}$ & $\begin{array}{l}\text { Los estudiantes pagan, pero no hay } \\
\text { fines lucrativos. } \\
\text { Pueden tener finalidad filantrópica o } \\
\text { no (la filantropía es la exención de } \\
\text { impuestos a cambio de becas para } \\
\text { estudiantes pobres, negros e } \\
\text { indígenas). }\end{array}$ \\
\hline
\end{tabular}


Los números de la enseñanza superior en Brasil muestran que en 2015 un 18,1\% de la población estaba frecuentando la enseñanza superior. La red privada responde por el 75,3\% de esos alumnos y las entidades estatales un 24,7\% (Brasil, 2016).

\section{¿Por qué las universidades comunitarias brasileñas necesitan cambiar su modelo de gestión?}

Las instituciones educacionales, sean ellas con o sin fines de lucro, cada vez más están buscando modelos de gestión que contemplen resultados y eficacia para la realización de su misión y de su visión. Al enfocar el modelo de gestión, tenemos la concepción de tal modelo como un proceso estructurado, interactivo y consolidado, sostenido por los instrumentos administrativos, estratégicos y por el estilo de los principales gestores de la organización - de desarrollar y manejar la administración estratégica, visando al incremento de los resultados y al crecimiento de la empresa (Acosta, et al., 2017, Oliveira, 2013).

Por razones históricas, la gestión de instituciones comunitarias se establecía en modelos menos complejos. Sin embargo, las demandas son derivadas de la complejidad del mundo contemporáneo. De esa manera, en la actualidad, se requieren nuevas posturas con relación a la gestión, según señala Drucker (2009), cuando explica que hace algunas décadas, la palabra "administración" estaba mal vista entre los involucrados en organizaciones sin fines de lucro, puesto que significaba "negocios". A la época, esas instituciones tenían orgullo en decir que estaban por encima de las preocupaciones con el mercado competitivo y de las sórdidas líneas de resultados. Sin embargo, el mismo autor admite que hoy día las entidades sin fines de lucro ya han entendido que necesitan una administración mucho más cuidadosa que las empresas, justamente porque les falta la disciplina que impone una estricta línea de resultados. Seguramente que esas instituciones siguen dedicándose a "hacer el bien", pero ya han percibido que buenas intenciones no sustituyen organización, liderazgo responsabilidad, desempeño y resultados.

Sousa (2011) asimismo presenta otros desafíos a los gestores de IES: hoy día, ellos están obligados a asumir nuevos compromisos, entre ellos, la responsabilidad de identificar que sus prácticas deberán atender a innúmeros desafíos con los que se enfrentan sus instituciones: sobrevivir, buscar nuevos caminos y crecer. Todo ello "enfrentando el aparecimiento de los grandes grupos formados a partir de fusiones y adquisiciones; la necesidad de superar las conductas y los modelos conservadores y ultrapasados de planeamiento para enfrentar la gran competición y para acompañar las exigencias del mundo del trabajo" (Souza, 2011,-- traducción nuestra).

En resumen, tradicionalmente muchas de las ICES se han pautado por conductas intuitivas, fruto de la existencia de demanda mayor que las vacantes ofrecidas. Eso era lo suficiente para garantizar los ingresos, y así, el mayor empeño se concentraba en sus objetivos socio educacionales (Moço, 2007, Dittadi, 2008). Sin embargo, a partir de los años 1990, el país pasa por un proceso de desreglamentación en la enseñanza superior (UFBA, 2011) y es afectado, a partir de los años 2000, por la globalización neoliberal de la universidad (Santos, 2011). Ello produce la reconfiguración del "mercado" de la educación brasileña, implicando que las instituciones busquen modelos gerenciales adecuados (Almeida, 2008). En este escenario, pronto las ICES han tenido que empezar un proceso de cambio y repensar sus modelos de gestión. Ello implicó atender a los nuevos desafíos contando con la adopción de herramientas de gestión, adecuadas a instituciones universitarias de base comunitaria. Este proceso involucra planear estrategias, estructurar procesos operacionales, medir el desempeño de las acciones y buscar los resultados deseados. Pasa asimismo por la adopción de métricas de desempeño, por tener un trabajo de más calidad en el equipo dirigente, mejor comunicación intra-organizacional y mejor gestión en los niveles operacionales (Kettunen, 2010).

Nuevos modelos de gestión transitan por una institución comunitaria sustentable, en las dimensiones administrativas y académicas. visando estabilidad económica y financiera, el desarrollo de la sociedad local y regional, y atención a la calidad ambiental (Hesselbarth y Schaltegger, 2014). Ello requiere la interacción con agentes externos a la Universidad (Fernández-Manzanal et al., 2015), envolvimiento de la comunidad académica, mejora de las comunicaciones externa e interna (Adomssen, 2013; Mader et al., 2013), consistencia entre acciones y comunicación y atención al campus de la institución (Jain et al., 2013).

Los esfuerzos en sentido de buscar modelos de gestión universitaria basados en prácticas sustentables implican que los gestores estén preparados para manejar los problemas que vendrán. Entre ellos, podemos subrayar: acciones desconectadas (Larrán y Andrades, 2015), apego a las prácticas tradicionales (Jabbour et al., 2013), limitaciones de capital, costos e informaciones (Maiorano y Savan, 2015), foco distinto entre las áreas administrativas y académicas en cuanto a las actividades de enseñanza, investigación y extensión, además de la oposición de la comunidad académica. Así, ante al nuevo escenario, las instituciones comunitarias sin fines de lucro han tenido que abdicar de una "posición cómoda" desde la cual ejercían su oficio, teniendo que repensar constantemente el perfeccionamiento del modelo de gestión y aun así 
continuar atendiendo su misión, credos y principios. Esas organizaciones necesitan adecuarse a un fenómeno que responde a mega tendencias asociadas con la evidente competitividad en la captación de alumnos, complejidad contextual donde están inseridas y necesidad de mayores niveles de eficiencia organizacional (Ganga y Burotto 2012, Ganga et al 2014).

\section{RESULTADOS Y DISCUSIÓN}

Basándonos en el resultado de la investigación, identificamos que el fenómeno relacionado con un proceso de perfeccionamiento del modelo de gestión de universidades comunitarias dialoga con lo que se verifica en la literatura. Las ICES son instituciones que se identifican con el legado histórico de la universidad (Clark, 2008), su capacidad de adaptación ante a nuevos escenarios (Baynaghi et. al., 2014), a los nuevos tiempos y a los nuevos paradigmas (Blowers, 1991), sus relaciones con el Estado, el mercado y con las comunidades (Cascio, 2018) y el perfeccionamiento de sus habilidades para formar profesionales y realizar investigación científica (Janeiro et. al., 2013). Trascendiendo sus raíces como universidad, las ICES tienen una responsabilidad social comunitaria (Moraes, 2006) diseminada en su cultura universitaria (Too y Bajracharya, 2015). Surgieron en Brasil de la necesidad de ofrecer servicios en el campo de la educación por la deficiencia o ausencia del Estado y precariedad de las instituciones públicas (Santos, 2011, Pinto, 2009).

A lo largo del tiempo, han tenido que demostrar modelos de gestión que contemplasen resultados y eficacia para la realización de su misión y de su visión, con base en sus raíces (Oliveira, 2013). Sin embargo, la reconfiguración del "mercado" de la educación superior brasileña (Almeida, 2008), ante a la desreglamentación de la enseñanza superior (UFBA, 2011; Santos, 2011), ha hecho que las ICES repiensen sus modelos intuitivos de gestión (Moço, 2007, Dittadi, 2008). Hoy día se presenta la necesidad de perfeccionar estos modelos con planeamiento, indicadores, desempeño y métricas (Kettunen, 2010) y asimismo buscar competitividad en la captación de alumnos y mayores niveles de eficiencia organizacional (Ganga y Burotto 2012, Ganga et al 2014). En lo que sigue se presentan los resultados relacionados con el perfeccionamiento del modelo de gestión en sus instituciones, como sigue. Han sido señalados cuatro puntos principales: la adecuación de estructuras y procesos; políticas de comunicación con el mercado, comunidades externa e interna; relación con autoridades gubernamentales y segmentos económicos, empresariales y sociales; articulaciones políticas y actitudes emprendedoras e innovadoras.

\section{Adecuación de estructuras y procesos}

En el entendimiento de los entrevistados y demás datos de la investigación, los modelos de gestión universitaria que sean adoptados por una institución comunitaria obligatoriamente deben compartir la misión, razón de existir y los principios que rigen la identidad comunitaria. Es decir, así como explican Oliveira (2013), Almeida Filho (2008), Santos (2011), Moço (2007) y Dittadi (2008), hay que conciliar la tradición y la innovación. En nuestro entendimiento, esa es una tarea compleja, pero es necesario adecuar procesos y estructuras a lo que exige la contemporaneidad, pero sin perder su identidad.

La realidad presenta ICES con grandes estructuras, con altos costos operacionales y con ociosidad de espacios físicos, principalmente en los turnos de la mañana y tarde. Esta realidad se justifica por atender a estudiantes trabajadores que también estudian, principalmente en el horario nocturno. De la misma forma, los procesos decisorios sufren por morosidad. La falta de visión estratégica y el no acompañamiento del tiempo del mercado hace que muchas ICEs presenten un tiempo decisorio no competitivo con el mercado. Dicha realidad exige profesionalización de la gestión para mayor competitividad y lucha por garantía de su lugar en el mercado educacional. A pesar las dificultades señaladas en la investigación, se evidencian iniciativas exitosas de adecuación de estructuras y definición de procesos operacionales en muchas ICES, con resultados satisfactorios en la atención a metas e indicadores esperados.

La identidad puede ser comprendida por educación que trasciende el saber y la formación profesional. Los gestores de las ICES lo entienden como una educación integral y humana (orientada a las personas), con bases en la cultura y en la investigación científica. A ello contribuye la no visión de lucro y la participación en programas de gobierno que beneficien a las comunidades académicas y del entorno social de la institución. Ser comunitario es tener una relación "distinta" con la comunidad. Es dar una contrapartida al desarrollo de la sociedad que es la base de la existencia de su universidad. Es participar en la vida de la comunidad, es ser un espacio acogedor, es formar egresados que cargan consigo la identidad institucional, es hacer parte de la historia local y de la región. Por fin, la gobernanza de una ICES, sus estructuras y procesos necesitan estar fundamentados en la inserción social, en el cuidado ambiental, mirando hacia la región, pero con una visión amplia y global. El compromiso se establece por el desarrollo de la sociedad, por la transparencia y visibilidad. 


\section{Políticas de comunicación con el mercado, comunidades externa e interna}

El modelo de gestión debe contemplar no solamente el desempeño organizacional, sino también un avance en la calidad académica y educación continua con bases en la cultura y en la investigación científica. Es decir, la no visión del lucro no puede ser confundida con la necesidad de sustentabilidad económica y financiera, puesto que hay que velar por el mantenimiento de la institución. Autores como Kettunen (2010), Ganga y Burotto (2012) y Ganga et al (2014) reafirman lo que hemos encontrado. Creemos que la ICES del siglo XXI deben superar la cultura de la queja por tener que adaptarse al mercado y dar un paso hacia más allá, mejorando la comunicación con su público externo e interno. Y eso se alcanza con políticas de comunicación insertadas en el proceso de profesionalización de la gestión.

La comunicación en una ICES aborda un público variado. Debe ser clara y concisa en transmitir al mercado (futuros alumnos) sus valores en cuanto a una educación integral y utilizar los principios de una institución comunitaria como un diferencial competitivo ante a la competencia principalmente. La comunicación debe trabajar asimismo constantemente para interaccionar con la comunidad académica (alumnos, profesores y funcionarios) y el público externo (comunidad, gobiernos, empresas y otras organizaciones en general). De forma amplia, las universidades que participaron en el estudio clasifican que la comunicación todavía persiste como un serio problema y desafío de las ICES. Si bien cuentan con tecnologías de la era de la información y muchas incluso disponiendo de medias universitarias como televisión, radio y periódico, los gestores son unísonos al reconocer la dificultad: "Por más que uno se comunique, la información no llega a todos los lugares y no alcanza a todos los públicos de interés". Reconocen la necesidad de mejorar las relaciones a través de una comunicación eficiente, pues la asignan como factor responsable de las relaciones de mercado, con la comunidad académica y el desarrollo regional y comunitario. Los datos refuerzan una frágil realidad vivida por las ICES en sus políticas de comunicación. Tanto interna como externamente, se evidencian acciones aisladas de endomarketing y marketing. Sin embargo, la ausencia de una política estratégica de comunicación resulta factor determinante para que la marca comunitaria pueda ganar mayor visibilidad.

\section{Relación con autoridades gubernamentales y segmentos económicos, empresariales y sociales}

El modelo de gestión tiene que estar sedimentado en procesos de articulaciones y relaciones público/privadas en nivel regional, nacional e internacional. De esta manera, podrá desarrollar relaciones que contemplen la necesaria participación en programas que involucren la participación en programas gubernamentales de financiación y proyectos de investigación. De acuerdo con Baynaghi et. al. (2014) y Cascio (2018), la universidad debe evolucionar junto con los nuevos tiempos y los nuevos paradigmas, perfeccionando su relación con el gobierno local, regional y nacional, así como con segmentos económicos y empresariales. De la misma forma, entendemos que el modelo de gestión institucional necesita contemplar políticas que visen a la evolución en la relación sustentable (y de doble mano) con la comunidad, para dar soporte al desarrollo de ambos. Las ICES son ejemplos de buenas prácticas de gestión en conjunto con otras universidades nacionales e internacionales, así como con gobiernos, empresas y comunidades. Ellas han entendido que están al servicio del desarrollo de sus comunidades locales y regionales y, para ello, necesitan invertir cada vez más en proyectos que se desarrollen en cooperación con las distintas fuerzas que mueven la economía y el desarrollo, desde la instancia local a la global.

Las prácticas de enseñanza en las ICES se establecen también en los proyectos comunitarios, como campo empírico de estudio. Tradicionalmente, una parte significativa de los proyectos sociales los sostenía la práctica de la filantropía y el presupuesto institucional. Actualmente, cambios en la legislación federal retiraron los recursos de la filantropía de los proyectos sociales, transfiriéndolos para concepciones de becas. De otra parte, recursos presupuestarios comprometen parte de las mensualidades de los alumnos en este sentido. Este es un ejemplo, entre otras situaciones que han estimulado acciones proactivas en cuanto a la sustentabilidad, a través de asociación con otras instituciones. Ello incluye capacidad para participar en pliegos públicos y privados, establecer convenios y acuerdos con autoridades municipales, asociaciones, comunidades y empresas privadas. Así, resulta en proyectos relacionados con la prestación de servicios públicos y privados, desarrollo de la innovación tecnológica y de emprendedurismo, a través de parques tecnológicos e incubadoras empresariales.

\section{Articulaciones políticas y actitudes emprendedoras e innovadoras.}

La universidad del siglo XXI necesita adoptar estrategias y buenas prácticas de marketing en la captación de alumnos. En este contexto, la identidad comunitaria debe servir como un diferencial competitivo por la calidad académica y la formación de ciudadanos éticos (Cascio 2018, Santos, 2011, Ganga et al, 2014). Los datos de la investigación están de acuerdo con estos autores, cuando explican que es necesario 
comprender que la gestión de las ICES requiere competencias y habilidades distintas, es decir, administrar una institución pública en cuanto a los fines y responsabilidades sociales, y privada en cuanto a los medios y fuentes de recursos exige profesionalización de la gestión. De esa manera, el uso de prácticas administrativas y aplicación de herramientas adecuadas para establecer indicadores y métricas demanda cualificación de los gestores. No más basta ser un buen profesor de carrera para ser un buen gestor. Las ICES se presentan en un movimiento sin precedentes en su articulación política, en sus planeamientos con foco en el emprendedurismo y en la innovación buscando dar respuestas significativas a su diferencial competitivo.

La calificación de los gestores de una universidad comunitaria implica, en la visión de los participantes en la investigación: habilidades como negociador en un ambiente complejo, que se relaciona con el mercado, el poder público, la academia y la sociedad. Implica desarrollar un papel de comprometimiento como persona pública, liderazgo y capacidad para resolución de problemas. Para ello, se exige profesionalización de la gestión para acciones relacionadas con planeamiento estratégico y organización de procesos administrativos y académicos. El gestor universitario necesita establecer el equilibrio entre las dimensiones relacionadas con la sustentabilidad y con las demandas académicas. Se añaden a la administración de una universidad no sólo conocimientos y habilidades administrativas, sino también competencias y habilidades de gestión académica (Villarroel y Bruna, 2017). Esto implica transitar por los modernos procesos pedagógicos, políticas y legislación de la educación superior. Se considera asimismo una actuación en las relaciones políticas en los campos educacionales y social.

\section{CONSIDERACIONES FINALES}

El artículo ha problematizado el perfeccionamiento de la gestión en universidades comunitarias brasileñas. Se ha abordado la necesidad de profesionalización de la gestión, con el uso de herramientas administrativas adecuadas a instituciones de enseñanza superior. Sin embargo, señalamos que estos procesos deben estar contenidos y en pie de igualdad con los fundamentos de una institución con base comunitaria.

El diálogo con distintos autores y los participantes en la investigación presentó la universidad comunitaria en el contexto del desarrollo histórico de la universidad moderna. Además, en el actual momento de la educación superior brasileña, se reconoce la importancia de las ICES y la necesidad de que estas instituciones se preparen para actuar en un mercado competitivo, buscando eficiencia organizacional. Los datos de la investigación traducen las ICES de Brasil en un momento histórico sin precedentes en la búsqueda por calificar sus prácticas de gestión en un contexto local, regional, nacional e internacional. A pesar de las dificultades para afirmarse en un mercado competitivo y frecuentemente agresivo, ya presentan resultados satisfactorios ante a los actuales retos que les impone la realidad.

Si bien la investigación tiene como albo la universidad brasileña, señalamos la contribución de este trabajo a otros contextos, puesto que, con el mundo globalizado, las mareas de cambios alcanzan indistintamente a la enseñanza universitaria y a la institución universidad en escala planetaria. En este sentido, consideramos que el debate sobre el tema y sobre buenas prácticas es necesario y muy saludable. Por fin, concluimos que el campo de la gestión ha sido una pauta de especial interés a los gestores de las IES ante a una economía de mercado que ha extendido su lógica al campo de la educación. En este escenario, las universidades se ven obligadas a buscar el perfeccionamiento de sus modelos de gestión para competir y continuar siendo una referencia para toda la sociedad.

\section{REFERENCIAS}

Acosta, L.A., F.A. Becerra y D. Jaramillo, Sistema de Información Estratégica para la Gestión Universitaria en la Universidad de Otavalo (Ecuador), doi: 10.4067/S0718-50062017000200011, Formación Universitaria, 10(2), 103-112 (2017)

Adomssen, M., Exploring universities' transformative potential for sustainability-bound learning in changing landscapes of knowledge communication, doi: https://goo.gl/6yoLMk, Journal of Cleaner Production, 49, 11-24 (2013)

Almeida Filho, J.C.P., Dimensões Comunicativas no ensino de línguas, Campinas, SP, Pontes (2008)

Alshuwaikhat, H.M. e I. Abubakar, An integrated approach to achieving campus sustainability: assessment of the current campus environmental management practices, doi: https://goo.gl/P2yqx2, J. of Cleaner Production, 16, 1777-1785 (2008)

Bardin, L., Análise de conteúdo, Lisboa, Edições, 70 (2006)

Brasil. Presidência da República, Constituição da República Federativa do Brasil, Brasília (1988)

Brasil. Presidência da República. Lei № 9.394, de 20 de dezembro de 1996. Estabelece as diretrizes e bases da educação nacional. Brasília: 20 de dezembro de (1996) 
Brasil. Lei № 12.711. Dispõe sobre o ingresso nas universidades federais e nas instituições federais de ensino técnico de nível médio e dá outras providências (2012)

Brasil. Lei № 12.881. Dispõe sobre a definição, qualificação, prerrogativas e finalidades das Instituições Comunitárias de Educação Superior - ICES, disciplina o Termo de Parceria e dá outras providências (2013)

Brasil. Instituto Nacional de Estudos e Pesquisas Educacionais Anísio Teixeira, INEP. Censo da Educação Superior 2016 (2006)

Blowers, A., Planning for a sustainable environment, 243, Earthscan, New York, USA (1991)

Cascio, W., Managing human resources, USA, McGraw-Hill Education (2018)

Clark, W., Academic charisma and the origins of the research university, USA, University of Chicago Press (2008)

Fernández-Manzanal, R., L.M. Serra y otros cinco autores, Environmental behaviours in initial professional development and their relationship with university education, doi: https://goo.gl/FMFyZ9, J. of Cleaner Production, 108, 830-840 (2015)

Fonif. Fórum Nacional das Instituições Filantrópicas. Pesquisa: a contrapartida do setor filantrópico para o Brasil. Brasília, Brasil (2016)

Foo, K.Y., A vision on the role of environmental higher education contributing to the sustainable development in Malaysia, doi: https://goo.gl/aFJvSo, Journal of Cleaner Production, 61, 6-12 (2013)

Ganga, F. y J. Burotto, Asimetrías de información entre agente y principal de las universidades chilenas, https://goo.gl/VZMrjE, ISSN: 0123-5923, Revista de Estudios Gerenciales, 28(122), 83-104 (2012)

Ganga, F., A. Cassinelli y otros tres autores, El concepto de eficiencia organizativa: una aproximación a lo universitario, https://goo.gl/Xztw2d, ISSN: 0719-5265, Revista Líder, 25, 126-150 (2014)

Goetsch, D.L. y S.B. Davis, Quality management for organizational excellence. 40, Pearson, Upper Saddle River, New Jersey, USA (2014)

Hesselbarth, C. y S. Schaltegger, Educating change agents for sustainability: Learnings from the first sustainability management master of business administration, doi: https://goo.gl/rmQLhw, J. of Cleaner Production, 62, 24-36 (2014)

Jabbour, C.J.C., J. Sarkis, A. Sousa y K. Govindan, Understanding the process of greening of Brazilian business schools, doi: https://goo.gl/GhqNeS, Journal of Cleaner Production, 61, 25-35 (2013)

Jain, S., P. Aggarwal, N. Sharma y P. Sharma, Fostering sustainability through education, research and practice: A case study of TERI University, doi: https://goo.gl/78ZP3b, Journal of Cleaner Production, 61, 20-24 (2013)

Kettunen, J., Strategic management in higher education: implementing strategic objectives, doi: https://goo.gl/6obTsT, Saarbrücken, Germany, 19(3), 207-2017 (2010)

Larrán, M. y J. Andrades, Determining factors of environmental education in Spanish universities, doi: https://goo.gl/CXHybU, International Journal of Sustainability in Higher Education, 16(2), 251-271 (2015)

Mader, M., C. Mader, F. Zimmermann y M. Diethart, Monitoring networking between higher education institutions and regional actors, doi: https://goo.gl/7FG2yS, Journal of Cleaner Production, 49, 105-113 (2013)

Maiorano, J., B. Savan, Barriers to energy efficiency and the uptake of green revolving funds in Canadian universities, doi: https://goo.gl/TvCnVT, International Journal of Sustainability in Higher Education, 16(2), 200-216 (2015)

Morgan, G., Reflections on images of organization and its implications for organization and environment, doi: https://goo.gl/JKK6LM, Organization \& Environment, 24(4), 459-478 (2011)

Müller-Christ, G., S. Sterling y otros cuatro autores, The role of campus, curriculum, and community in higher education for sustainable development: A conference report, doi: https://goo.gl/w5pDSk, J. of Cleaner Production, 62, 134-137 (2014)

Oliveira, D.P.R., Administração Estratégica na prática: a competitividade para administrar o futuro das empresas, 320, Atlas, São Paulo, Brasil (2013)

Pinto, R.A., Universidade Comunitária e Avaliação Institucional: o caso das Universidades comunitárias gaúchas, https://goo.gl/EPkq9E, ISSN: 1982-5765, Avaliação, São Paulo, 14(1), 185-215 (2009)

Santos, B.S., A universidade no século XXI: para uma reforma democrática e emancipatória da universidade, 89, Cortez, São Paulo, Brasil (2011)

Santos, B.S., If God were a human rights activist, 152, Stanford University Press, Standford, USA (2015)

Stake, R.E., Investigación con estudio de casos, Madrid, Ediciones Morata (1998)

Tan, H., S. Chen, Q. Shi y L. Wang, Development of green campus in China, doi: https://goo.gl/p1tYSt, Journal of Cleaner Production, 64, 646-653 (2014)

Villarroel, V. A. y D.V. Bruna, Competencias Pedagógicas que Caracterizan a un Docente Universitario de Excelencia: Un Estudio de Caso que Incorpora la Perspectiva de Docentes y Estudiantes, doi: 10.4067/S0718-50062017000400008, Formación Universitaria, 10(4), 75-96 (2017)

Wals, A.E.J., Sustainability in higher education in the context of the DESD: A review of learning and institutionalization processes, doi: https://goo.gl/PEctfh, Journal of Cleaner Production, 62, 8-15 (2014) 
